\title{
JURISPRUDENCIA AMBIENTAL EN LA COMUNIDAD DE MADRID (PRIMER SEMESTRE 2020)
}

\author{
DANIEL B. ENTRENA RUIZ \\ Profesor Contratado Doctor \\ Universidad Carlos III de Madrid \\ daniel.entrena@uc3m.es
}


Sumario: 1. Espacios Naturales Protegidos. Montes de Utilidad Pública de titularidad de Entidad Local Histórica (Comunidad de la Ciudad y Tierra de Segovia). Admisibilidad del esquí de fondo. Sentencia del Tribunal Superior de Justicia de Madrid, Sección 4a, 27/2020 de 22 de enero de 2020. 2. Protección del suelo. Instalaciones deportivas, recreativas y culturales en zona de transición de espacio natural protegido; uso admitido en suelo rústico, previo trámite de calificación urbanística. Obligatoria motivación de la denegación. Sentencia del Tribunal Superior de Justicia de Madrid 40/2020, de 12 de febrero. 3. Protección del suelo. Actividades de ocio en suelo no urbanizable o urbanizable no programado. Necesidad de prohibición expresa por planeamiento urbanístico para denegar su implantación. Sentencia de Tribunal Superior de Justicia de Madrid 20/2020, de 6 de febrero. 4. Residuos y Suelos Contaminados. Falta de competencia municipal para sancionar vertidos ilegales respecto aquellos residuos cuya competencia no ha asumido. Origen: Sentencia del Tribunal Superior de Justicia de Madrid 31/2020, de 6 de febrero. 5. Contaminación acústica por actividades de ocio. Declaración de Zona de Protección Acústica. Adecuación de las mediciones acústicas para su adopción. Sentencia del Tribunal Superior de Justicia de Madrid 16/2020, de 6 de febrero.

\section{1.- ESPACIOS NATURALES PROTEGIDOS. MONTES DE UTILIDAD PÚBLICA DE TITULARIDAD DE ENTIDAD LOCAL HISTÓRICA (COMUNIDAD DE LA CIUDAD Y TIERRA DE SEGOVIA). ADMISIBILIDAD DEL ESQUÍ DE FONDO. SENTENCIA DEL TRIBUNAL SUPERIOR DE JUSTICIA DE MADRID, SECCIÓN 4a , 27/2020 DE 22 DE ENERO DE 2020.}

La Sentencia con la que comenzamos el recopilatorio jurisprudencial de este semestre en la Comunidad de Madrid, resuelve el recurso contenciosoadministrativo interpuesto por la Comunidad de la Comunidad de la Ciudad y Tierra de Segovia. Este tipo de entidades fueron conformadas durante la Baja Edad Media para ordenar los procesos de repoblación y, organizadas entorno a divisiones administrativas denominadas Sexmos, aglutinaba diversos municipios actuales en torno al de la ciudad de Segovia, tanto de esta provincia como de Ávila y Madrid.

La pretensión de dicha Entidad Local en el recurso formulado era el reconocimiento de la existencia de vía de hecho en la ocupación ilegal de Monte de Utilidad Pública (MUP) no 113 "La Cinta-Peñalara", del que aquélla es titular, como consecuencia de la ocupación de la Pista de esquí de Fondo de Cotos, en un $80 \%$ de su trazado, cuya gestión venía siendo desarrollada por la Comunidad de Madrid, quien 
organizaba anualmente eventos en ella, sin ningún tipo de participación ni compensación.

En atención a esa supuesta ocupación ilegal la Entidad Local reclamaba una indemnización los daños y perjuicios, que incorporase un $25 \%$ de penalización, más los intereses devengados desde el año 2000, cuando comenzó a funcionar la pista de esquí de fondo.

Por su parte, la CAM interesaba la desestimación del recurso invocando que el terreno afectado la consideración de Monte de Utilidad Pública y está registrado en el catálogo de Montes de Utilidad Pública de la Comunidad de Madrid por lo que, de conformidad con la legislación de montes, se trata de un bien de dominio público cuya gestión corresponde a dicha Comunidad Autónomo.

Pues bien, la Sala va a desestimar la pretensión solicitada por la mencionada Entidad Local no como consecuencia de la determinación de la titularidad del espacio afectado por la pista de esquí, como podría suponerse, sino por la de su gestión conforme a la legislación de montes estatal y autonómica.

De este modo, conforme a la Ley 43/2003, de 21 de noviembre, de Montes, "Las entidades locales, en el marco de la legislación básica del Estado y de la legislación de las comunidades autónomas, ejercen las competencias siguientes: a) La gestión de los montes de su titularidad no incluidos en el Catálogo de Montes de Utilidad Pública; b) La gestión de los montes catalogados de su titularidad, cuando así se disponga y en la forma que se disponga en la legislación forestal de la comunidad autónoma".

Por su parte, la Ley 16/1995, de 4 de mayo, Forestal y de Protección de la Naturaleza de la Comunidad de Madrid, señala en su artículo 12.1 que "el Catálogo de Montes de Utilidad Pública de la Comunidad de Madrid es un registro público de carácter administrativo en el que se incluirán los montes que con anterioridad a esta Ley hubieran sido declarados de Utilidad Pública y los que lo sean en lo sucesivo." Por su parte, el artículo 22 de esta Ley establece: "Corresponde a la Comunidad de 
Madrid la administración y gestión directa de los montes o terrenos forestales siguientes: (...) c) Los montes catalogados de Utilidad Pública cuyo titular es una entidad local".

De este régimen concluye la Sala que "De conformidad con este régimen legal, resulta que el monte objeto de este proceso es un monte de dominio público o demanial, cuya administración y gestión directa corresponde a la Comunidad de Madrid".

De forma adicional, resulta que el Monte Peñalara-La Cinta se encuentra una parte dentro del Parque Natural de la Cumbre, Circo y Lagunas de Peñalara y otra dentro de su Zona Periférica de Protección, siendo por ello aplicable el Decreto 178/2002, de 14 de noviembre, que aprueba el Plan de Ordenación de los Recursos Naturales, y que admite con carácter general los usos turístico-recreativos con fines contemplativos, educativos y de disciplinas deportivas que no constituyan un riesgo para la conservación de los valores naturales del Parque Natural; permite, en particular, el excursionismo y senderismo y establece que "el esquí de montaña y de fondo tendrán la consideración de uso tradicional a los efectos de este PORN".

De todo ello concluye la Sentencia referenciada que si "para que una actuación de la Administración Pública pueda ser considerada realizada en vía de hecho, es preciso que estemos ante una actuación material de la Administración, que carezca de la más mínima cobertura jurídica, debe considerarse que conforme a lo expuesto, todas las actuaciones pueden considerarse realizadas en uso de las legítimas competencias de gestión que a la CAM atribuye la normativa aplicable al monte y de la planificación del mismo, lo que conforme a lo invocado por la demandada permite descartar que esa actuación sobre la pista de esquí pueda considerarse como una ocupación en vía de hecho".

Por tanto, de lo actuado la Sala concluye que, al no existir vía de hecho, tampoco existe derecho de indemnización alguna, y que lo pretendido por la demandante era la obtención de algún beneficio económico que debería eventualmente haberse plasmado el correspondiente convenio de colaboración. 
2-. PROTECCIÓN DEL SUELO. INSTALACIONES DEPORTIVAS, RECREATIVAS Y CULTURALES EN ZONA DE TRANSICIÓN DE ESPACIO NATURAL PROTEGIDO; USO ADMITIDO EN SUELO RÚSTICO, PREVIO TRÁMITE DE CALIFICACIÓN URBANÍSTICA. OBLIGATORIA MOTIVACIÓN DE LA DENEGACIÓN. SENTENCIA DEL TRIBUNAL SUPERIOR DE JUSTICIA DE MADRID 40/2020, DE 12 DE FEBRERO-

La Congregación de los hermanos de la instrucción cristiana, hermanos menesianos, recurre la Orden 2.933/2017, de 4 de octubre de 2017, del Consejero de Medio Ambiente, Administración Local y Ordenación del Territorio de la Comunidad de Madrid que se deniega la calificación urbanística para la implantación de instalaciones deportivas, recreativas y culturales, en la Finca Los Frailes, del término municipal de Madrid.

La razón de dicha denegación estuvo motivada en que que en virtud de lo dispuesto en los informes emitidos por el Director Conservador del Parque Regional de la Cuenca Alta del Manzanares se trataba de usos incompatibles con la legislación del Parque Regional de la Cuenca Alta del Manzanares (Zona T).

En contraste que dicha limitación, en el PGOU de la ciudad de Madrid dicho uso sí estaba admitido en el área de transición del Monte de El Pardo, donde se encontraba la Finca, si bien al tratarse de suelo rústico era exigible el trámite de calificación urbanística por la Comunidad Autónoma, además de someterse el proyecto a evaluación de impacto ambiental.

Por esta razón, debido a la incompatibilidad del uso conforme al Plan de Ordenación de los Recursos Naturales del Parque Regional, la Administración Autonómica resolvió denegar la solicitud de calificación ambiental sin ni siquiera someterlo al preceptivo trámite de evaluación de impacto ambiental simplificado, incluso a pesar de que pudiera ser un uso urbanísticamente admitido.

Sin embargo, la resolución autonómica fue efectuada sin tener en cuenta que los informes emitidos debieron ser emitidos por el Pleno del Patronato del Parque 
Regional de la Cuenca Alta del Manzanares, de conformidad con la Ley 1/1985 del Parque Regional de la Cuenca Alta del Manzanares, además de que confundían la concreta ubicación del Proyecto y no examinaba su compatibilidad con los fines protectores asignados a la Zona de Transición Ecológica.

Por estos motivo considera la Sala que la Orden autonómica impugnada adolecía de la necesaria motivación, considerándola nula de pleno Derecho, con retroacción de actuaciones al momento inmediatamente anterior al solicitar la emisión del preceptivo informe al Pleno del Patronato del Parque Regional de la Cuenca Alta del Manzanares u órgano administrativo que le hubiere sustituido.

\section{3.- PROTECCIÓN DEL SUELO. ACTIVIDADES DE OCIO EN SUELO NO URBANIZABLE O URBANIZABLE NO PROGRAMADO. NECESIDAD DE PROHIBICIÓN EXPRESA POR PLANEAMIENTO URBANÍSTICO PARA DENEGAR SU IMPLANTACIÓN. SENTENCIA DE TRIBUNAL SUPERIOR DE JUSTICIA DE MADRID 20/2020, DE 6 DE FEBRERO.}

La Sentencia estima el recurso contencioso-administrativo contra la denegación de la calificación urbanística solicitada a la Comunidad Autónoma para implantar la actividad referida. Un uso que en Madrid fue admitido por la Ley 8/2012 de 28 de diciembre, de medidas fiscales y administrativas de la Comunidad de Madrid, cuyo artículo 9 admitía la expresa implantación en suelo no urbanizable de usos vinculados a la celebración de actos sociales y eventos familiares en edificaciones existentes. Indica así el referido precepto:

\footnotetext{
"Tendrán la consideración de usos permitidos y autorizables que favorecen el desarrollo rural sostenible, los usos vinculados a la celebración de actos sociales y eventos familiares de especial singularidad, así como los usos caninos y ecuestres, en edificaciones existentes que puedan ser habilitadas a esos exclusivos fines, que se ubiquen en suelo no urbanizable con cualquier protección y suelo urbanizable no sectorizado.
} 
Los usos previstos en el párrafo anterior se considerarán, en todo caso, compatibles con las normas de protección establecidas en la legislación sectorial de la Comunidad de Madrid.

Estos usos podrán ser autorizados mediante el procedimiento de calificación urbanística previsto en la Ley 9/2001, de 17 de julio, del Suelo de la Comunidad de Madrid, y deberán contar en todo caso con la preceptiva licencia municipal. Se exceptúan los supuestos en que el planeamiento municipal expresamente los prohíba".

Para resolver el recurso, el Tribunal va a centrar su atención en el contenido del planeamiento urbanístico del municipio donde se pretendía implantar el uso solicitado.

Mientras la Comunidad de Madrid deducía que las normas subsidiarias de Valdetorres de Jarama, sí prohibían dicho uso, los demandantes entendían lo contrario, puesto que no estaba prohibido expresamente.

En particular, el artículo 8.10.2 de las normas urbanísticas establecía un régimen de protección de terrenos para la explotación de cultivos o policultivos agrícolas, huertos y en general suelos agrícolas de alta potencialidad, "que por su rentabilidad y su adecuada y homogénea disposición conjunta merecen ser destinados de manera intensiva y exclusiva a dicho uso, constituyendo, en sí mismos la base económica al municipio".

En este tipo de terrenos se prohibía, en primer lugar, "cualquier acción encaminada al cambio de uso por otros de distinta índole". Y únicamente podían producirse calificaciones urbanísticas cuando tuviesen por finalidad

“a) Construcciones e instalaciones de apoyo a los usos propios que sirvan a unidades funcionales y productivas desarrolladas íntegramente sobre terrenos pertenecientes a esa categoría de suelo, u otra igualmente restrictiva, e imprescindibles para el desarrollo de la actividad (art. 53, apdo. a) $(\ldots)$ 
b) Actividades indispensables para el establecimiento, funcionamiento conservación o mantenimiento de las redes infraestructurales básicas o servicios públicos, siempre que se demostrase la inexistencia de una ubicación o trazado alternativo que pudiese evitar esta clase de suelo sin comprometer otros espacios de mayor valor ambiental (art.23 apdo. d)"

Como puede comprobarse, el planeamiento sujetaba los usos admisibles con calificación urbanística autonómica a concretas construcciones, instalaciones y actividades, en general vinculadas bien a la actividad agrícola bien al funcionamiento de los servicios públicos.

En estas previsiones se amparó la Comunidad de Madrid para denegar la calificación ambiental.

Pues bien, tras su examen, la Sala anula la denegación realizada al considerar que no existía una prohibición expresa del uso solicitado y que, de admitir una prohibición extensiva, se estaría contraviniendo el régimen estipulado en la Ley autonómica 8/2012, de 28 de diciembre:

"dicho uso no está expresamente prohibido en el artículo 8.10.2 de las Normas Urbanísticas ya que por uso expresamente prohibido y como ya hemos dicho en nuestra anterior sentencia de 13 de marzo de 2019, recurso 46472016, debemos entender como uso expresamente prohibido aquel que nominalmente se prohíbe en dichas normas, no pudiendo acogerse la interpretación relativa a que como el planeamiento municipal indica que los usos permitidos son exclusivamente los relacionados con la actividad agrícola, ello determina que únicamente sea posible la autorización de tales usos, con exclusión de todos los demás. $\mathrm{Y}$ no se puede acoger dicha interpretación pues supondría dejar vacío de contenido el artículo 9 de la Ley 8/2012, norma con la que el legislador autonómico ha pretendido considerar compatible con el desarrollo rural sostenible el uso solicitado, en suelo no urbanizable con cualquier protección, salvo que "expresamente" el planeamiento municipal lo prohíba, lo que no es el caso. Los únicos usos 
expresamente prohibidos en el artículo 8.10.2 de las Normas Urbanísticas son el uso de vivienda, la realización de actividades extractivas y la implantación de vertederos del ámbito señalado, y la ganadería intensiva no relacionada con el aprovechamiento de pastos en la propia parcela".

Esta doctrina supone que si el planeamiento urbanístico quiere limitar determinados usos en suelo no urbanizable de protección, en la Comunidad de Madrid debe concretarse si admite los contenidos en la Ley 8/2012, de 28 de diciembre, pues de otro modo se consideran directamente admitidos y compatibles.

En la actualidad, esa Ley continúa plenamente vigente, siendo uno de los aspectos más controvertidos en el ámbito urbanístico de la Comunidad de Madrid por las implicaciones ambientales que representa.

\section{4.- RESIDUOS Y SUELOS CONTAMINADOS. FALTA DE COMPETENCIA MUNICIPAL PARA SANCIONAR VERTIDOS ILEGALES EN ZEC Y ZEPA, RESPECTO AQUELLOS RESIDUOS CUYA COMPETENCIA NO HA ASUMIDO. ORIGEN: SENTENCIA DEL TRIBUNAL SUPERIOR DE JUSTICIA DE MADRID 31/2020, DE 6 DE FEBRERO.}

Esta resolución judicial examina en apelación la conformidad a Derecho de la sentencia de 17 de mayo de 2018, dictada por el Juzgado de lo ContenciosoAdministrativo no6 de Madrid, en el Procedimiento Ordinario número 164/2017, que había anulado lo la sanción de 80.000 euros impuesta por un municipio madrileño (Daganzo) a los titulares de un camping por la comisión de cuatro infracciones de la Ley 22/2011, de 28 de julio, de Residuos y Suelos Contaminados, sustancialmente por el abandono sistemático de forma continuada, de vertidos de todo tipo en sus proximidades; concretamente, según informe técnico de medio ambiente, residuos de construcción y demolición, restos de podas, maderas, plásticos, colchones, cartones y envases de plástico (denuncia del Seprona); muebles y enseres, frigoríficos, lavadoras, televisiones, residuos de construcción y demolición, restos de poda, colchones, restos de envases de plástico, latas y bricks, residuos quemados. Se da la circunstancia de que el camping estaba dentro de la Zona de 
Especial Conservación (ZEC) "Cuenca de los Ríos Jarama y Henares" y la Zona de Especial Protección de Aves (ZEPA) "Estepas cerealistas de los Ríos Jarama y Henares".

El elemento neurálgico que se plantea es si el Ayuntamiento tenía competencias suficientes para sancionar dicha conducta conforme a las previsiones de la Ley de Residuos y Suelos Contaminados, teniendo en cuenta que no prestaba servicio de recogida transporte y tratamiento de los residuos comerciales generados en el camping por considerar que no era una competencia obligatoria municipal.

Pues bien, tanto el Juzgado primero como la Sala de lo Contencioso-Administrativo posteriormente van a considerar que carecía de dicha competencia, en el segundo caso por dos motivos distintos.

Respecto a los residuos comerciales porque, de la Ley de Residuos y Suelos Contaminados, "parece desprenderse (art.12.5) que los Ayuntamientos "pueden" asumir competencias respecto de los residuos comerciales, y éste no era el caso". Por ello, en atención a lo previsto en el artículo 49.3 de la referida Ley, la consecuencia es que no podía sancionar dicha conducta:

"En el supuesto de abandono, vertido o eliminación incontrolados de los residuos cuya recogida y gestión corresponde a las Entidades Locales de acuerdo con el artículo 12.5, así como en el de su entrega sin cumplir las condiciones previstas en las ordenanzas locales, la potestad sancionadora corresponderá a los titulares de las Entidades Locales"

Un precepto que fue modificado, por cierto, mediante el Real Decreto-Ley 17/2012, de 4 de mayo (luego Ley 11/2012, de 19 de diciembre), porque originariamente no establecía ese nexo tan claro entre la gestión y la sanción aparejada: "En el supuesto de abandono, vertido o eliminación incontrolados de residuos, así como de su entrega sin cumplir las condiciones previstas en las ordenanzas locales, la potestad sancionadora corresponderá a los titulares de las Entidades Locales". 
Pero, además, considera la Sala que, con independencia de que los residuos fueran comerciales o domésticos, "el Ayuntamiento no tenía potestad para sancionar el vertido, (...) porque, si eran residuos domésticos, no había ejercido sus competencias de gestión de residuos respecto de los del camping. Aunque en éste último caso, deberíamos precisar, se trataría más bien de un supuesto de falta de culpabilidad de la recurrente en los hechos sancionados".

La Sentencia de apelación no explicita por qué no concurría ese elemento de culpabilidad según la vertida en la Instancia, pero el razonamiento parece ser que al no cumplir sus obligaciones el Ayuntamiento, difícilmente podía exigir a los titulares del camping que evitaran la realización de los vertidos incontrolados.

\section{5.- CONTAMINACIÓN ACÚSTICA POR ACTIVIDADES DE OCIO. DECLARACIÓN DE ZONA DE PROTECCIÓN ACÚSTICA. ADECUACIÓN DE LAS MEDICIONES ACÚSTICAS PARA SU ADOPCIÓN. SENTENCIA DEL TRIBUNAL SUPERIOR DE JUSTICIA DE MADRID 16/2020, DE 6 DE FEBRERO.}

La última de las Sentencias que deseamos resaltar en este número es singular, porque denota que en ocasiones la Administración Pública continúa sin ser altamente beligerante contra la contaminación acústica, pese a los evidentes efectos que provoca sobre la salud humana y las múltiples condenas recaídas contra su pasividad, al amparo a su vez del derecho fundamental a la intimidad domiciliaria.

Se trata de la comunidad de propietarios de un inmueble del centro de la ciudad de Madrid, que se dirige contra el Acuerdo del Pleno del Ayuntamiento de Madrid, de 28 de junio de 2017, por el que se declara Zona de Protección Acústica Especial el Barrio de Gaztambide, y se aprueba su Plan Zonal Específico.

La pretensión de la entidad demandante era que dictase sentencia por la que se "acuerde dejar sin efecto la clasificación de la manzana ocupada por el edificio de la Comunidad demandante como Zona de Contaminación Acústica Baja y 
otorgando la que resulte de la prueba pericial solicitada en el Segundo Otrosí Digo o, subsidiariamente de lo anterior, acuerde que por la Administración demandada se lleve a cabo una nueva campaña de mediciones con los medios adecuados que incluyan al menos tres puntos de medición en el interior de la Comunidad demandante en una de las tres zonas descritas en el Hecho Segundo de esta demanda".

La normativa del Plan Zonal Específico, con el objeto de disminuir el nivel de contaminación acústica, establecía entre otras cosas un régimen limitativo de implantación o modificación de las actividades, en atención a tres zonas de protección acústica de intensidad diferenciada, con medidas correctoras diferentes para su recuperación: zona de contaminación acústica alta (zona que presenta una superación de los objetivos de calidad acústica en el descriptor Ln, igual o superior a $10 \mathrm{dBA}$ en el ambiente exterior, artículo 7), zona de contaminación acústica moderada (zona que presenta una superación de los objetivos de calidad acústica en el descriptor $\mathrm{Ln}$, igual o superior a $5 \mathrm{dBA}$ e inferior a $10 \mathrm{dBA}$ en el ambiente exterior, artículo 10) y zona de contaminación acústica baja (zona que presenta una superación de los objetivos de calidad acústica en el descriptor Ln, menor de 5 dBA en el ambiente exterior, artículo 13).

Como indica en el suplico de la demanda, recién reproducido, la actora sostenía la indebida clasificación del edificio en la Zona de Contaminación Acústica Baja por la Normativa de la ZPAE de Gaztambide, poniendo de relieve la concentración de locales de ocio existente en el lugar. A su parecer, la medición de la contaminación acústica existente había sido insuficiente, pues tan solo se determinó un punto de medición para la medición de la zona en dos cotas en altura, con edificios de 13 plantas.

En contraste, el Ayuntamiento de Madrid sostenía la legalidad de la inclusión en la zona de baja contaminación acústica del inmueble pues las mediciones realizadas mostraban niveles de ruido elevados exclusivamente durante el periodo nocturno de días festivos y de sus vísperas, y sin embargo, los días de diario, en especial los laborables, no se superaban en el edificio los objetivos de calidad acústica previstos 
en el ZPAE; por ello, resultaba procedente adoptar medidas concretas para acabar con los niveles de ruido provocados por la concentración de locales de ocio de la zona, medidas para acabar con el "botellón", así como con el ruido producido por el tráfico rodado que circula por la zona. Unas medidas que ya habían sido adoptadas por el Ayuntamiento.

Para solucionar el conflicto, la Sala examina en primer lugar el régimen contenido en el punto 3.4.1 del Anexo IV del Real Decreto 1367/2007, de 19 de octubre, según el cual la Evaluación de los índices de ruido referentes a objetivos de calidad acústica en áreas acústicas exige realizar "una evaluación preliminar mediante mediciones en continuo durante al menos 24 horas, correspondientes a los episodios acústicamente más significativos, atendiendo a la fuente sonora que tenga mayor contribución en los ambientes sonoros del área acústica".

Sin embargo, según fue acreditado en autos, dicha medición preliminar no fue efectuada, decidiendo los servicios técnicos municipales emplear un único equipo de medición de ruido en un concreto punto, considerando que sería suficiente para caracterizar acústicamente el recinto interior del edificio, pero sin llegar a motivar por qué se optó por ello, pese a las concretas particularidades concurrentes de la zona (elevada concentración de locales de negocio, terrazas, topografía, servidumbre de paso público, ...).

Por ese motivo, la Sala entiende pertinente acoger el suplico subsidiario de la demanda, debiendo el Ayuntamiento de Madrid llevar a cabo una nueva campaña de mediciones con los medios adecuados, que incluyan "al menos un punto de medición en cada una de las tres zonas señaladas.

En atención a dichas mediciones debía entonces el Ayuntamiento de Madrid establecer la calificación (zona de protección acústica baja, moderada o alta) que correspondiese, según lo previsto al respecto en el Acuerdo del Pleno del Ayuntamiento de Madrid, de 28 de junio de 2017, por el que se declara Zona de 
Protección Acústica Especial el barrio de Gaztambide, y aprueba su Plan Zonal Específico, Distrito de Chamberí. 